\title{
Diffractive jets production in pp-collisions
}

\author{
D.Yu. Ivanov*, V.M. Braun ${ }^{\dagger}$ and A. Schäfer ${ }^{\dagger}$ \\ ${ }^{*}$ Sobolev Institute of Mathematics and Novosibirsk State University, 630090 Novosibirsk, Russia \\ ${ }^{\dagger}$ Institut für Theoretische Physik, Universität Regensburg, D-93040 Regensburg, Germany
}

\begin{abstract}
We consider the exclusive diffractive dissociation of a proton into three jets with large transverse momenta in the double-logarithmic approximation of perturbative QCD. This process is sensitive to the proton unintegrated gluon distribution at small $\mathrm{x}$ and to the proton light-cone distribution amplitudes. According to our estimates, an observation of such processes in the early runs at LHC is feasible for jet transverse momenta of the order of $5 \mathrm{GeV}$.
\end{abstract}

Keywords: hard diffraction, jets

PACS: $12.38 . \mathrm{Bx}, 12.39 . \mathrm{St}, 13.87 .-\mathrm{a}$

1. We explore the possibility to observe hard exclusive diffractive dissociation of a proton into three hard jets in proton-proton collisions

$$
p\left(p_{1}\right)+p\left(p_{2}\right) \rightarrow j e t\left(q_{1}\right)+j e t\left(q_{2}\right)+j e t\left(q_{3}\right)+p\left(p_{2}^{\prime}\right) .
$$

In this process one proton stays intact and the other one dissociates into a system of three hard jets separated by a large rapidity gap from the recoil proton, see Fig. 1.

Note that we are interested in exclusive three-jet production which constitutes a small fraction of the inclusive single diffraction cross section. The exclusive and inclusive mechanisms have different final state topologies and can be distinguished experimentally. A characteristic quantity is e.g. the ratio $R_{\text {jets }}$ of the three-jet mass to the total invariant mass of the system produced in the diffractive interaction. Exclusive production corresponds to the region where $R_{\text {jets }}$ is close to unity. This strategy was used recently at the Tevatron [1] where central exclusive dijet production, $p \bar{p} \rightarrow p+j e t+j e t+\bar{p}$, in double-Pomeron collisions was measured for the first time.

Exclusive dijet production in the central region has much in common with the exclusive Higgs boson production process, $p \bar{p} \rightarrow p+H+\bar{p}$. In [2] it was argued that studies of exclusive dijet production and other diffractive processes at the early data runs of the LHC can provide valuable checks of the different components of the formalism. Indeed, this was the main motivation for Tevatron experiment. The exclusive 3-jets production in single diffraction (1) offers another interesting example since factorization of hard and soft interactions in this case is less complicated. In particular, the fluctuation of a proton projectile into a state with small transverse size, which is the underlining mechanism for (1), suppresses secondary soft interactions that may fill the rapidity gap. Thus one can get an access to the gluon distribution at small $x$ in a cleaner environment, having no problems with gap survival probability and factorization breaking that introduce major conceptual theoretical uncertainties in the calculations of diffractive Higgs production.

Our approach to exclusive three-jet production derives from experience with coherent pion diffraction dissociation into a pair of jets with large transverse momenta which was 


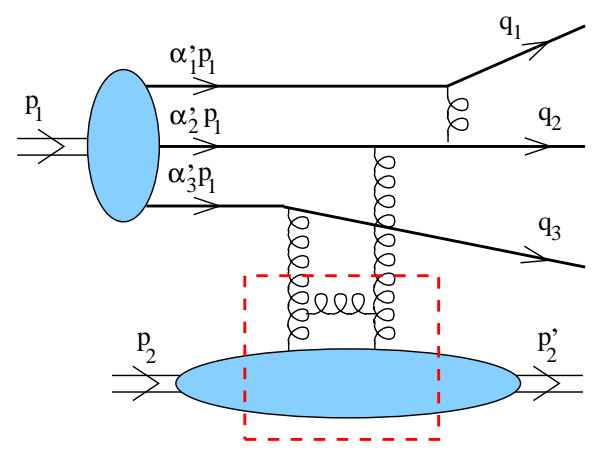

FIGURE 1. Proton dissociation into three jets. The unintegrated gluon distribution includes the hard gluon exchange as indicated by the dashed square.

measured by the E791 collaboration [3, 4]. The qualitative features of the E791 data have confirmed some earlier theoretical predictions [5, 6, 7]: a strong A-dependence which is a signature for color transparency, and a $\sim 1 / q_{\perp}^{8}$ dependence on the jet transverse momentum. These features suggest that the relevant transverse size of the pion $r_{\perp}$ remains small, of the order of the inverse transverse momenta of the jets $r_{\perp} \sim 1 / q_{\perp}$.

We have shown [8, 9] that collinear factorization is violated in dijet production due to pinching of singularities between soft gluon (and quark) interactions in the initial and final state. However, the nonfactorizable contribution is suppressed compared to the leading contribution by a logarithm of energy so that in the double logarithmic approximation $\ln q_{\perp}^{2} \ln s / q_{\perp}^{2}$ collinear factorization is restored. Moreover, to this accuracy hard gluon exchange can be "hidden" in the unintegrated gluon distribution $\mathscr{F}\left(x, q_{\perp}\right)$. Thus, in the true diffraction limit, for very large energies, hard exclusive dijet production can be considered as a probe of the hard component of the pomeron. The same interpretation was suggested earlier in [10] within the $k_{t}$ factorization framework. The double logarithmic approximation turns out to be insufficient for the energy range of the E791 experiment, but might be adequate for the LHC. Here we present an estimate for the cross section for the reaction (11) based on the generalization of these ideas.

2. At leading order the jets are formed by the three valence quarks of the proton, see Fig. 1. We require that all three jets have large transverse momenta which requires at least two hard gluon exchanges. One of them can be effectively included in the highmomentum component of the unintegrated gluon density (the bottom blob) as indicated schematically by the dashed square, but the second one has to be added explicitly since the hard pomeron only couples to two of the three quarks of the proton.

Our notation for the momenta is explained in Fig. 1. We neglect power corrections in transverse momenta of the jets and also proton and jet masses so that $p_{1}^{2}=p_{2}^{2}=p_{2}^{\prime 2}=$ $q_{1}^{2}=q_{2}^{2}=q_{3}^{2}=0$. The jet momenta are decomposed in terms of momenta of the initial particles

$$
q_{k}=\alpha_{k} p_{1}+\beta_{k} p_{2}+q_{k \perp}, \quad k=1,2,3 .
$$


The three-jet invariant mass is given by

$$
M^{2}=\left(q_{1}+q_{2}+q_{3}\right)^{2}=\frac{\vec{q}_{1 \perp}^{2}}{\alpha_{1}}+\frac{\vec{q}_{2 \perp}^{2}}{\alpha_{2}}+\frac{\vec{q}_{3 \perp}^{2}}{\alpha_{3}}, \quad \zeta=\frac{M^{2}}{s}=\beta_{1}+\beta_{2}+\beta_{3} .
$$

where $s=\left(p_{1}+p_{2}\right)^{2}=2 p_{1} \cdot p_{2}$ is the invariant energy. Assuming that the relevant jet transverse momenta are of the order of $5 \mathrm{GeV}$, the typical values of the $\zeta$ variable at LHC are in the range $\zeta \sim 10^{-6} \div 10^{-5}$.

The relevant Feynman diagrams can be divided into three groups which differ by the attachments of the $t$-channel gluons to the quark lines. Accordingly, we have three different contributions to the amplitude (for the details see [11]):

$$
\begin{aligned}
\mathscr{M} & =-i 2^{7} \pi^{5} s \alpha_{s}^{2}\left[\frac{e^{i j k}\left(\frac{1+N}{N}\right)^{2}}{4 N !\left(N^{2}-1\right)}\right] \int D \alpha^{\prime} \times\left(\mathscr{L}_{1} \frac{\delta\left(\alpha_{1}-\alpha_{1}^{\prime}\right)}{q_{1 \perp}^{4}} \mathscr{F}\left(\zeta, q_{1 \perp}\right)\right. \\
& \left.+\mathscr{L}_{2} \frac{\delta\left(\alpha_{2}-\alpha_{2}^{\prime}\right)}{q_{2 \perp}^{4}} \mathscr{F}\left(\zeta, q_{2 \perp}\right)+\mathscr{L}_{3} \frac{\delta\left(\alpha_{3}-\alpha_{3}^{\prime}\right)}{q_{3 \perp}^{4}} \mathscr{F}\left(\zeta, q_{3 \perp}\right)\right),
\end{aligned}
$$

where $\int D \alpha^{\prime}=\int_{0}^{1} d \alpha_{1}^{\prime} d \alpha_{2}^{\prime} d \alpha_{3}^{\prime} \delta\left(1-\sum \alpha_{i}^{\prime}\right)$ corresponds to the integration over the quark momentum fractions in the incident proton, $e^{i j k}$ describes the color state of the final quarks, $N=3$ is the number of colors. The dimensionless quantities $\mathscr{L}_{i}$ are expressed in terms of the leading-twist light-cone nucleon distribution amplitudes.

3. The differential cross section can be written as

$$
d \sigma=\frac{|\mathscr{M}|^{2}}{2^{5}(2 \pi)^{8} s^{2}} \frac{d \alpha_{1} d \alpha_{2} d \alpha_{3} \delta\left(1-\alpha_{1}-\alpha_{2}-\alpha_{3}\right)}{\alpha_{1} \alpha_{2} \alpha_{3}} d^{2} \vec{q}_{1} d^{2} \vec{q}_{2} d t d \phi_{t}
$$

where $t=\left(p_{2}-p_{2}^{\prime}\right)^{2}$ is the Mandelstam $t$ variable of the $p p$ scattering and $\phi_{t}$ is the azimuthal angle of the final state proton. In our kinematics, for large transverse momenta of the jets and small $t$, one can neglect effects of azimuthal correlations between the jets and the final proton. Hence $d \phi_{t}$ integration is trivial and gives a factor $2 \pi$. For the $t$ dependence we assume a simple exponential form, $d \sigma / d t \sim e^{b t}$, and use $b \sim 4 \div 5 \mathrm{GeV}^{2}$ for the slope parameter which is a typical value which describes HERA data for hard exclusive processes: DVCS and vector meson electroproduction at large $Q^{2}$. Thus, the integration over the proton recoil variables gives a factor $\int d t d \phi_{t} \rightarrow \frac{2 \pi}{b}$.

Since our calculation is only done to double logarithmic accuracy, we use the simplest model for the unintegrated gluon distribution as given by the logarithmic derivative of the usual gluon parton distribution $x g\left(x, Q^{2}\right)$

$$
\mathscr{F}\left(x, q_{\perp}^{2}\right)=\frac{\partial}{\partial \ln q_{\perp}^{2}} x g\left(x, q_{\perp}^{2}\right) .
$$

In our numerical estimates we use the CTEQ6L leading-order gluon distribution [12].

The integration over the phase space of the three jets was done numerically, restricting the longitudinal momentum fractions to the region

$$
0.1 \leq \alpha_{1}, \alpha_{2}, \alpha_{3} \leq 0.8
$$


and requiring that the transverse momentum of each jet is larger than a given value $q_{0}=q_{\perp \text {,min }}$. For $q_{0}=5 \mathrm{GeV}$ we obtain for the integrated three-jet cross section at the LHC energies

$$
\sigma_{3-\text { jets }}^{\mathrm{LHC}}=4 \mathrm{pb} \cdot\left(\frac{f_{N}\left(q_{0}\right)}{4.7 \cdot 10^{-3} \mathrm{GeV}^{2}}\right)^{2}\left(\frac{\alpha_{s}\left(q_{0}\right)}{0.21}\right)^{4}\left(\frac{5 \mathrm{GeV}}{q_{0}}\right)^{9} .
$$

Assuming the integrated luminosity for the first LHC runs in the range $100 \mathrm{pb}^{-1}$ to $1 \mathrm{fb}^{-1}$ an observation of this process at LHC seems to be feasible. Note that the effective power $\sigma \sim 1 / q_{0}^{9}$ (fitted in the $q_{0}=3 \div 8 \mathrm{GeV}$ range) is somewhat stronger than the naive power counting prediction $\sigma \sim 1 / q_{0}^{8}$. This effect is due to the strong $\zeta$ dependence of the unintegrated gluon distribution: larger values of $q_{0}$ imply larger invariant masses $M^{2}$ of the three-jet system (3) and consequently larger $\zeta=M^{2} / s$. The sizeable cross section for $q_{0}=5 \mathrm{GeV}$ is in fact an implication of the expected rise of the LO gluon distribution more than two times as $\zeta$ is decreasing by roughly a factor of 50 when going from Tevatron to LHC. The existing parameterizations of the LO gluon distribution at $\zeta \sim 10^{-6}$ differ from each other by $\sim 30 \%$. The unintegrated gluon distribution (6) enters as a square in the prediction for the cross section, therefore, the study of exclusive three-jet events at LHC may provide a valuable constraint for the gluon distribution at small momentum fractions.

A comparison of the three-jet exclusive production at LHC and the Tevatron can be especially illuminating in this respect since other uncertainties do not have significant impact on the energy dependence. For Tevatron kinematics, assuming the value $q_{\perp \min }=$ $3 \mathrm{GeV}$, our estimate for the cross section (fitted in the range $q_{0}=2 \div 4.5 \mathrm{GeV}$ ) is

$$
\sigma_{3-\text { jets }}^{\text {Tevatron }}=50 \mathrm{pb} \cdot\left(\frac{f_{N}\left(q_{0}\right)}{4.7 \cdot 10^{-3} \mathrm{GeV}^{2}}\right)^{2}\left(\frac{\alpha_{s}\left(q_{0}\right)}{0.255}\right)^{4}\left(\frac{3 \mathrm{GeV}}{q_{0}}\right)^{9} .
$$

\section{ACKNOWLEDGMENTS}

D. I. was supported in part by the grants RFBR-08-02-00334-a and NSh-1027.2008.2.

\section{REFERENCES}

1. T. Aaltonen et al. [CDF Run II Collaboration], Phys. Rev. D 77 (2008) 052004.

2. V. A. Khoze, A. D. Martin and M. G. Ryskin, Eur. Phys. J. C 55 (2008) 363.

3. E. M. Aitala et al. [E791 Collaboration], Phys. Rev. Lett. 86 (2001) 4768.

4. E. M. Aitala et al. [E791 Collaboration], Phys. Rev. Lett. 86 (2001) 4773.

5. S. F. King, A. Donnachie and J. Randa, Nucl. Phys. B 167 (1980) 98; J. Randa, Phys. Rev. D 22 (1980) 1583.

6. G. Bertsch, S. J. Brodsky, A. S. Goldhaber and J. F. Gunion, Phys. Rev. Lett. 47 (1981) 297.

7. L. Frankfurt, G. A. Miller and M. Strikman, Phys. Lett. B 304 (1993) 1.

8. V. M. Braun, D. Yu. Ivanov, A. Schäfer and L. Szymanowski, Phys. Lett. B 509 (2001) 43.

9. V. M. Braun, D. Yu. Ivanov, A. Schäfer and L. Szymanowski, Nucl. Phys. B 638 (2002) 111.

10. N. N. Nikolaev, W. Schäfer and G. Schwiete, Phys. Rev. D 63 (2001) 014020.

11. V. M. Braun, D. Yu. Ivanov and A. Schafer, Phys. Lett. B 666 (2008) 245.

12. J. Pumplin, D. R. Stump, J. Huston, H. L. Lai, P. Nadolsky and W. K. Tung, JHEP 0207 (2002) 012. 